\title{
ERRATUM
}

\section{Erratum to: Lactate-guided resuscitation saves lives: no}

Xavier Monnet ${ }^{1 *}$ and Anthony Delaney ${ }^{2}$

C 2016 Springer-Verlag Berlin Heidelberg and ESICM

\section{Erratum to: Intensive Care Med (2016) 42:470-471 DOI 10.1007/s0134-016-4235-5}

This editorial published in the March 2016 issue of Intensive Care Medicine forms part of an editorial debate on the topic of lactate-guided resuscitation.

Unfortunately, a third author was mistakenly added during the publication process: Dr. Amber Barnato took no part in the writing process and should have not been listed among the authors.

The correct authorship is as shown here. The ICM editorial office offers its apologies to the readers for this mistake.

\section{Electronic supplementary material}

The online version of this article (doi:10.1007/s00134-016-4554-6) contains supplementary material, which is available to authorized users.

\section{Author details}

${ }^{1}$ Medical Intensive Care Unit, Inserm UMR S_999, Hôpitaux universitaires Paris-Sud, Université Paris-Sud, Le Kremllin-Bicetre, France. ${ }^{2}$ Northern Clinical School, Sydney Medical School, University of Sydney, Sydney, New SouthWales, Australia.

Published online: 22 September 2016 